\title{
Effects of hyperoxia on vascular tone in animal models: systematic review and meta-analysis
}

Bob Smit ${ }^{*}$ (D), Yvo M. Smulders², Etto C. Eringa ${ }^{3}$, Heleen M. Oudemans - van Straaten ${ }^{1}$, Armand R. J. Girbes ${ }^{1}$, Kimberley E. Wever ${ }^{4}$, Carlijn R. Hooijmans ${ }^{4}$ and Angelique M. E. Spoelstra - de Man ${ }^{1}$

\begin{abstract}
Background: Arterial hyperoxia may induce vasoconstriction and reduce cardiac output, which is particularly undesirable in patients who already have compromised perfusion of vital organs. Due to the inaccessibility of vital organs in humans, vasoconstrictive effects of hyperoxia have primarily been studied in animal models. However, the results of these studies vary substantially. Here, we investigate the variation in magnitude of the hyperoxia effect among studies and explore possible sources of heterogeneity, such as vascular region and animal species.

Method: Pubmed and Embase were searched for eligible studies up to November 2017. In vivo and ex vivo animal studies reporting on vascular tone changes induced by local or systemic normobaric hyperoxia were included. Experiments with co-interventions (e.g. disease or endothelium removal) or studies focusing on lung, brain or fetal vasculature or the ductus arteriosus were not included. We extracted data pertaining to species, vascular region, blood vessel characteristics and method of hyperoxia induction. Overall effect sizes were estimated with a standardized mean difference (SMD) random effects model.
\end{abstract}

Results: We identified a total of 60 studies, which reported data on 67 in vivo and 18 ex vivo experiments. In the in vivo studies, hyperoxia caused vasoconstriction with an SMD of $-1.42(95 \% \mathrm{Cl}-1.65$ to -1.19$)$. Ex vivo, the overall effect size was SMD $-0.56(95 \% \mathrm{Cl}-1.09$ to -0.03$)$. Between-study heterogeneity $\left(l^{2}\right)$ was high for in vivo $(72 \%$, $95 \% \mathrm{Cl} 62$ to $85 \%$ ) and ex vivo studies (86\%, 95\% Cl 78 to 98\%). In vivo, in comparison to the overall effect size, hyperoxic vasoconstriction was less pronounced in the intestines and skin $(P=0.03)$ but enhanced in the cremaster muscle region $(P<0.001)$. Increased constriction was seen in vessels $15-25 \mu \mathrm{m}$ in diameter. Hyperoxic constriction appeared to be directly proportional to oxygen concentration. For ex vivo studies, heterogeneity could not be explained with subgroup analysis.

Conclusion: The effect of hyperoxia on vascular tone is substantially higher in vivo than ex vivo. The magnitude of the constriction is most pronounced in vessels $\sim 15-25 \mu \mathrm{m}$ in diameter and is proportional to the level of hyperoxia. Relatively increased constriction was seen in muscle vasculature, while reduced constriction was seen in the skin and intestines.

Keywords: Hyperoxia, Microcirculation, Vasoconstriction, Meta-analysis

\footnotetext{
* Correspondence: b.smit@vumc.nl

'Department of Intensive Care, VU University Medical Center, De Boelelaan

1117, 1007, MB, Amsterdam, The Netherlands

Full list of author information is available at the end of the article
}

(c) The Author(s). 2018 Open Access This article is distributed under the terms of the Creative Commons Attribution 4.0 International License (http://creativecommons.org/licenses/by/4.0/), which permits unrestricted use, distribution, and reproduction in any medium, provided you give appropriate credit to the original author(s) and the source, provide a link to the Creative Commons license, and indicate if changes were made. The Creative Commons Public Domain Dedication waiver (http://creativecommons.org/publicdomain/zero/1.0/) applies to the data made available in this article, unless otherwise stated. 


\section{Background}

Superfluous oxygen supplementation in the acute, perioperative and intensive care setting frequently leads to arterial hyperoxia [1-3] and is associated not only with pulmonary side effects, but also with substantial acute hemodynamic changes. Hyperoxia can increase systemic vascular resistance due to (systemic) vasoconstriction and reduce cardiac output.

Although increased systemic vascular resistance indicates vasoconstriction, it does not inform in which organ the constriction occurs or at which location in the arterial tree. It is important for patient care to know whether constriction occurs equally or preferentially in organs. For instance, it may be argued that restriction of blood flow to resting skeletal muscle is less consequential than constriction in active cardiac muscle or organs like the liver and kidney. In animals, a hyperoxia-induced redistribution of blood flow to the kidney and splanchnic area has been observed, suggesting that vessels in these areas respond differently to hyperoxia [4-6].

Vascular diameter measurements in clinically relevant organs (e.g. visceral organs) often require surgical intervention. In humans, observations of hyperoxia-induced changes in vessel diameter in the microcirculation are therefore limited to superficial vascular beds such as the retina [7-9] and the sublingual vascular bed [10]. These organs are possibly not representative of hyperoxia-related vessel diameter changes in critical organs. Consequently, the effects of oxygen on vascular tone in deeper organs have been studied predominantly in animal models, using intravital microscopy (in vivo) and myography of isolated arteries (ex vivo). However, the results of these studies vary tremendously. Raising the oxygen tension to hyperoxic levels could lead to vasoconstriction [11-13], no effect [14-16] or even vasodilation [17, 18]. The causes of this heterogeneity are unclear, but might include differences between species, study methodology, vascular beds and/or the location of the hyperoxia sensor (e.g. the vessel wall or extravascular tissue). Identifying the source(s) of heterogeneity may contribute to a better understanding of the mechanism(s) involved in hyperoxic vasoconstriction [19].

With this systematic review and meta-analysis, we aim to provide an overview of the characteristics, quality and outcomes of all animal studies on the effect of hyperoxia on arteriolar tone, both in vivo and ex vivo. We also investigate the variation in magnitude of the hyperoxia effect among studies and explore sources of heterogeneity.

\section{Methods}

This review is reported according to the preferred reporting items for systematic reviews and meta-analyses (PRISMA) guidelines [20]. The review methodology was specified in advance and documented using The Systematic Review Centre for Laboratory Animal
Experimentation (SYRCLE) systematic review protocol for animal intervention studies ([21] and Additional file 1) and was registered on www.syrcle.nl on 22-02-2017. The protocol describes separate strategies for in vivo and ex vivo studies. However, due to considerable overlap and to enhance readability, we have combined the description of the review methodology where possible. The combined review question was: what is the effect of normobaric hyperoxia or hyperoxic superfusion on vascular tone of in vivo and ex vivo arteries and arterioles, in comparison to normoxia or normoxic superfusion?

\section{Amendments to the review protocol}

To assess the risk of bias in studies, we planned to use the SYRCLE risk of bias tool. This tool has several items that relate to studies in which separate intervention and control groups are used. In such experiments, animal housing and animal selection may have a significant impact on outcomes. However, the studies included in this review (both in vivo and ex vivo) were all short-term pretest-posttest designs, performed in the same animal/ vessels (e.g. no control groups). As a consequence, the SYRCLE risk of bias tool was not suitable for this review and was substituted by a customized quality checklist (see "Quality assessment" section). Due to the large variation in the size of the arteries investigated in the in vivo experiments, we added artery size post hoc as a possible source of heterogeneity (see "Data analysis").

\section{Search strategy}

We searched the databases Pubmed and Embase for articles published from inception up to 22 November 2017. The search strategy included various terms for vasoconstriction, vasodilation and hyperoxia. These categories were then combined with the AND operator and filtered for animal studies with the SYRCLE animal filter [22, 23]. We did not apply any language or date restrictions. Additional file 1 shows the search strategies used. We also checked the reference lists of included studies and relevant reviews for additional articles on hyperoxia that were not identified in the initial search.

\section{Selection of studies}

Studies identified by the initial search were subjected to 3-phase screening, performed by two investigators (BS and AS). In the first phase, titles were screened to exclude studies not related to blood vessels or hyperoxia. In the second phase, titles and abstracts of articles were screened for eligibility based on the following predefined inclusion criteria: (1) controlled studies with repeated measures involving (2) adult healthy animals or isolated arteries or arterioles from adult healthy animals, (3) short-term normobaric, normocapnic hyperoxia in comparison with a normoxic control (see "Oxygenation 
definitions" for an explanation), (4) vessel diameter or tension measurements reported. In case of doubt, the full text of the publication was evaluated. Disagreements were resolved by discussion (BS and AS). In phase 3, full-text articles were assessed for final inclusion in more detail, based on the following exclusion criteria: (1) case reports, reviews and studies without an intervention, (2) disease models or models focusing on the vascular beds of the lung, brain or retina, the fetal vasculature or the ductus arteriosus, (3) studies without a focus on vasoreactivity, (4) vessels preconditioned by hypoxia, an intervention in the experimental design other than oxygen (e.g. addition of $\mathrm{CO}_{2}$, endothelium removal) or long term hyperoxia, (5) none of our predefined outcome measures were reported. In particular, the brain was excluded from analysis because it is known to possess extensive mechanisms for the regulation of its perfusion, therefore making it unsuitable for inclusion in meta-analysis of other more comparable vasculatures. Similarly, we excluded studies in non-healthy animals, to reduce anticipated heterogeneity because of altered regulation of perfusion during states of disease.

\section{Oxygenation definitions}

For the in vivo studies, when the intervention was applied systemically, the measurements while the animal was breathing a gas with $20-21 \% \mathrm{O}_{2}$ were considered as normoxic. Consequently, measurements during the inhalation of a gas with an oxygen content $>21 \%$ were recorded as hyperoxic. For in vivo studies where hyperoxia was established locally through superfusion, normoxia was defined as the state where tissue was superfused with a physiologic salt solution (PSS) equilibrated with $0-5 \% \mathrm{O}_{2}$. With these concentrations, supply of oxygen to tissue by the PSS is limited as much as possible and occurs predominantly through the microcirculation. In these studies, hyperoxia was defined as superfusion with a PSS with an oxygen concentration of $>5 \%$.

In ex vivo studies, partial pressure of oxygen $\left(\mathrm{PO}_{2}\right)$ is directly comparable to partial pressure of oxygen in arterial blood $\left(\mathrm{P}_{\mathrm{a}} \mathrm{O}_{2}\right)$. Hence, the lower limit of normoxia was defined as PSS equilibrated with at least $7 \% \mathrm{O}_{2}$, which yields a $\mathrm{PO}_{2}$ of $\sim 55 \mathrm{mmHg}$. Because ex vivo oxygen exposures are often much higher than attainable in vivo (e.g. $100 \% \mathrm{O}_{2}$ ex vivo gives a $\mathrm{PO}_{2}$ of $\sim 760 \mathrm{mmHg}$, while inhalation of pure oxygen gives a $\mathrm{P}_{\mathrm{a}} \mathrm{O}_{2}$ of approximately $350 \mathrm{mmHg}$ ), we used measurements at the lowest non-hypoxic oxygen exposure as the "normoxic" state and any exposures above as "hyperoxic".

\section{Data extraction}

From each article we extracted data on the species, strain, age, weight and sex of the animals used, type of anesthetic used, method and level of oxygen exposure, type of preparation/measurement setup, buffers used, descriptions of variability in preparations and oxygen dose-response relations. Reports on a change in vascular tone upon exposure to hyperoxia were extracted as "experiments" (e). Sometimes an experiment was not supported by data (e.g. data not shown). Experiments with data were extracted as "data sets" (k). These data sets include the number of animals/vessels used, mean and standard deviation or standard error of the vessel diameter (or equivalent, e.g. tension), or change from baseline data, during normoxic and hyperoxic exposures. Data not reported numerically were extracted from graphs with a digital ruler if possible. Because of incomplete outcome data in five studies, we contacted the corresponding author by email to request additional information. We received a response from one author, who was unable to supply the missing data. If a study reported multiple responses from similar vessels in separate groups (e.g. different unique control groups), then only the data from the group with the largest number of paired vessels were used in this meta-analysis. If there was uncertainty about the number of observations (e.g. the number of observations was reported as a range), the lowest value was used to calculate a conservative estimate of the standard error. All data were extracted by one author (BS) and then randomly checked by a second (AS).

\section{Quality assessment}

The quality of studies was assessed using a tailor-made checklist containing ten items regarding blinding, preparation quality, verification of intervention efficacy, selective outcome reporting, description of animals and arteries, power analysis, ethical approval, conflicts of interest and peer review. For a list of the criteria used, see Additional file 2.

\section{Data analysis}

All calculations were made using RStudio v1.1.383 and the "metafor" and "dosresmeta" package (Integrated Development for R. RStudio, Inc., Boston, USA). Graphs were made using GraphPad Prism 7.0 (GraphPad Software, Inc., La Jolla, USA) or RStudio. Effect sizes are presented as the standardized mean difference (SMD) for series of experiments involving paired comparisons as proposed by Gibbons [24]. All statistics are reported with their 95\% confidence interval. An estimation of the variance of the SMD was done for a one group design with repeated measures [24]. Missing $\mathrm{SD}_{\text {difference }}$ values were calculated assuming correlation between measurements of 0.7 [25]. A sensitivity analysis using higher and lower correlation coefficients was conducted to test the robustness of our results. Effect estimates of the maximal hyperoxic exposure were pooled using a random 
effects model. Heterogeneity was assessed by the $I^{2}$ statistic and is reported with its $95 \%$ confidence interval [26].

As a priori sources of heterogeneity we considered animal species, sex, vascular bed and method of hyperoxia exposure for in vivo studies. For ex vivo studies, we considered species, sex, tissue origin of the vessel, vessel type (systemic or resistance) and the presence of flow. We added baseline artery diameter post hoc as a possible source of heterogeneity for in vivo studies. The relation between effect size and artery diameter was explored through restricted cubic spline modeling, because it does not require any assumption on the type of relation between the two variables (e.g linear, sigmoidal etc.) [27]. The Akaike information criteria (AIC) was used to determine the optimal number of knots and their position [28]. For each subgroup a minimum of five data sets, from three unique studies, had to be present. Sources of heterogeneity were investigated using meta-regression, by first performing an overall test for interaction, and if the $P$ value was $<0.05$, pairwise comparisons between subgroups were made to detect further subgroup interactions.
To correct for multiple testing, $P$ values were adjusted with the Holms-Bonferroni method.

The likelihood of publication bias was assessed using the trim and fill method [29]. Because SE-based precision estimates cause distortion of SMD funnel plots we used $1 / \sqrt{ } \mathrm{n}$ as the precision estimate in the trim and fill analysis [30].

\section{Results}

\section{Search and study selection}

A flow chart of the study selection process is shown in Fig. 1. After exclusion based on title and abstract, we identified 319 articles investigating the relation between oxygen and vascular tone: 261 studies were excluded based on predefined exclusion criteria. From the 60 included studies, 42 were performed in live animals and 18 studies used isolated arteries and arterioles. One study performed both eligible in vivo and ex vivo experiments [31]. Studies were performed between 1974 and 2017 and the majority (64\%) was published between 1980 and 2000 .

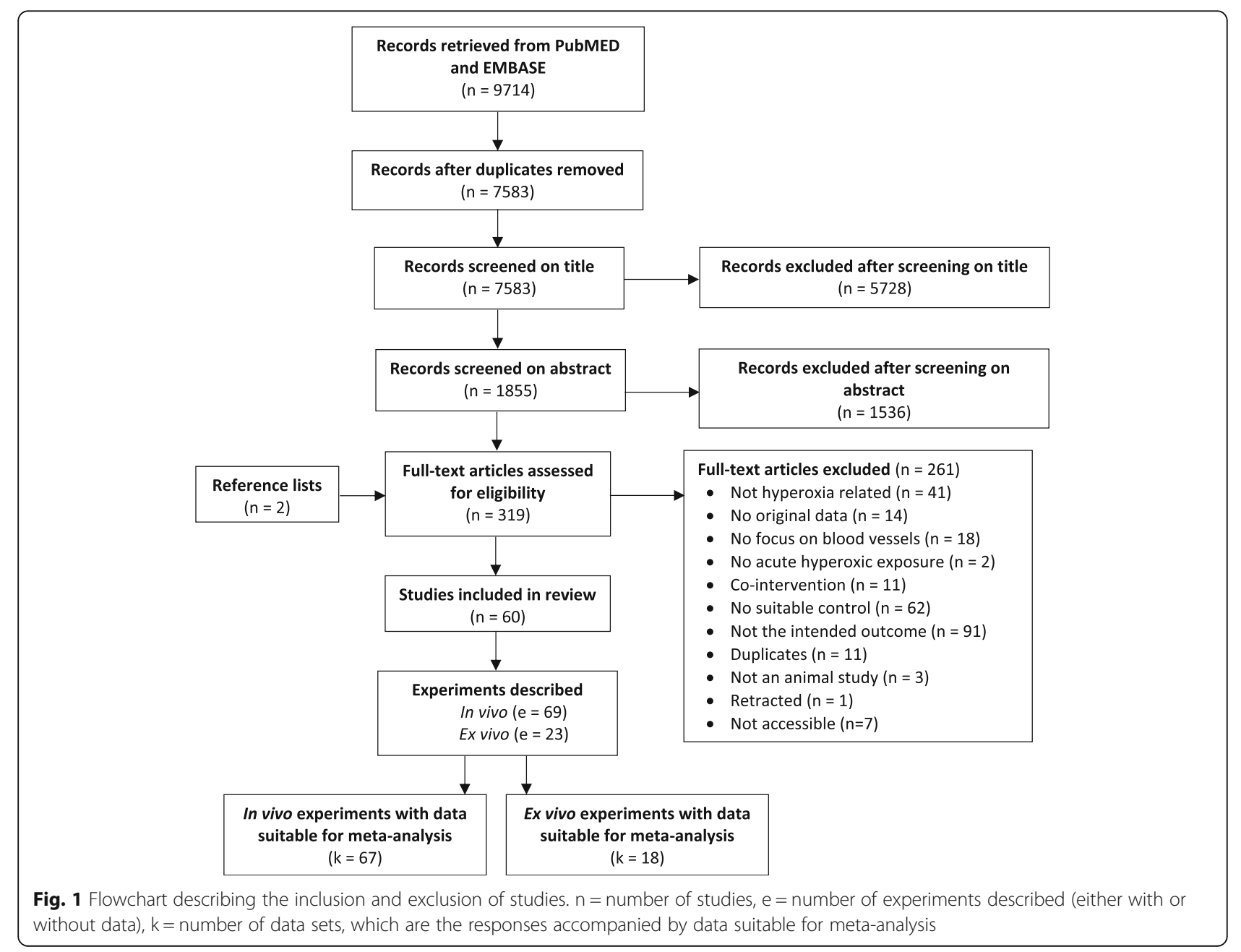


Characteristics of the included studies are presented in Tables 1 (in vivo studies) and 2 (ex vivo studies).

\section{Study characteristics In vivo studies}

The 42 in vivo studies were carried out in rats $(n=19)$, hamsters $(n=17)$, mice $(n=3)$, rabbits $(n=3)$ and cats $(n=1)$. In one study both rats and hamsters were used [32]. Most studies exclusively used male animals $(n=27)$. In one study both male and female animals were used, in one only females were used and in the remaining studies the sex of animals was not reported. Rats were between 5 and 18 weeks of age and weighed 75-413 g (range of study averages). Ages were not reported for other animals. Hamsters weighed 55-200 g, mice 24-35 g, rabbits 3$4 \mathrm{~kg}$ and cats $0.9-1.5 \mathrm{~kg}$. In most studies the animals were anesthetized with pentobarbital $(n=28$, used predominantly in rats and hamsters). Other anesthetics were thiopental [33-35], chloralose [36, 37], urethane [37, 38], ketamine [39] or a mixture of droperidol, midazolam and fentanyl [40-42]. In some studies skinfold windows were applied so that the microcirculation could be observed without anesthesia [14, 43-45].

Each study reported 1-6 experiments in which blood vessel diameter was observed while hyperoxia was induced. Combined, a total of 69 experiments (e) were described in these studies, which included measurements on arterioles with a median diameter of $20 \mu \mathrm{m}$ (interquartile range $14-34 \mu \mathrm{m}, 1$ st to 6 th order arterioles) in the cremaster $(e=20)$, cheek pouch $(e=16)$, dorsal skin $(e=10)$, sartorius muscle $(e=6)$, mesoappendix $(e=6)$, spinotrapezius muscle $(e=4)$, ear $(e=2)$, ileum $(e=2)$, retractor muscle $(e=1)$, sciatic nerve $(e=1)$ and the sublingual microcirculation $(e=1)$. The area of interest was superfused with a physiologic salt solution, equilibrated with $0 \%$ or $5 \% \mathrm{O}_{2}$ (normoxia, $e=52$ and $e=4$, respectively) and subsequently exposed to $10 \%$ (hyperoxia, $e=9), 21 \%(e=46)$ or $95 \% \mathrm{O}_{2}(e=1)$. In 13 experiments, blood vessels were observed through a surgically implanted window ( $e=7$, in the cheek pouch or the ear) or non-invasively by a hand-held device $(e=1)$, while hyperoxia was induced systemically by increasing the fraction of oxygen inspired by the animal $(e=13)$.

\section{Ex vivo studies}

In the 18 ex vivo studies, isolated arteries from rats $(n=7)$, pigs $(n=4)$, rabbits $(n=3)$, cows $(n=2)$, mice $(n=1)$ and sheep $(\mathrm{n}=1)$ were used. In 10 studies male animals were used and in one study animals of either sex were used [15]: in the remaining 7 studies animal sex was not reported. Rats were 6-15 weeks old and weighed 200-500 g. Pigs were about 4 months old and weighed 70-100 kg. Mice were 10-12 weeks old and weighed
$25 \mathrm{~g}$ on average. The age and weight of cows and sheep were not reported.

In these studies, 23 experiments were described. The maximal diameters of the vessels ranged between 100 and $5000 \mu \mathrm{m}$ and they were exposed to hyperoxia in muscle chambers $(e=10)$, wire myographs $(e=5)$ and pressure myographs $(e=8)$. Both conduit (thoracic aorta, femoral artery, coronary artery) and resistance arteries (gracilis arteriole, cremaster arteriole) were investigated. All studies used physiological salt solutions with comparable contents. After isolation, the blood vessels in some studies spontaneously developed tone $[13,16,18$, $31,46-50]$. Isolated vessels that did not develop tone were left unconstricted [17, 51-53] or were constricted to $\sim 40-50 \%$ of their baseline diameter with norepinephrine $[16,54,55]$, phenylephrine [56], histamine [15], potassium chloride $[15,18,55]$, thromboxane (U46199) [53], serotonin (5-HT) [53] or prostaglandin F2 $\alpha$ [53]. Vessels were exposed to normoxia by gassing the surrounding buffer with $10-21 \%$ oxygen. In one study, $40 \%$ oxygen was used in the control group [15]. Hyperoxia was induced by changing the gas supply to $21 \%$ or $95 \%$ $\mathrm{O}_{2}$. In 11 experiments, the authors measured the $\mathrm{PO}_{2}$ of the solutions, which ranged from 78 to $160 \mathrm{mmHg}$ for normoxia and 280-617 $\mathrm{mmHg}$ for hyperoxia.

\section{Quality assessment}

See Fig. 2 for an overview of the quality assessment. Overall, the reporting of key quality indicators was poor in both in vivo and ex vivo studies. Only 2 out of 60 studies (3\%) reported blinding of any phase of the experiment; these two cases were in vivo studies where the outcome assessor was blind to the experimental groups. Quality checks of the preparation were not described in $66 \%$ of the studies and $62 \%$ of the studies did not report whether and how the intervention was verified (e.g. by measuring $\mathrm{PO}_{2}$ levels). Similarly, the reporting of other preparation conditions (temperature, $\mathrm{CO}_{2}$ and $\mathrm{pH}$ ) was insufficient in $79 \%$ and $78 \%$ of the studies, respectively. Adequate descriptions of relevant details about the animals (e.g. sex, number of animals used), or vessels (diameters, selection) were lacking in $70 \%$ and $78 \%$ of the studies, respectively. Only one study performed a power analysis. The majority of studies (66\%) were published in peer-reviewed journals, however, for 4 studies it was unclear whether these were peer-reviewed. Only 6 studies $(10 \%)$ provided a conflict of interest statement and only 24 studies (40\%) mentioned approval by an ethical board.

\section{Descriptive synthesis of outcomes In vivo effect of hyperoxia on vascular tone}

According to the effects reported by the original authors (Table 1), raising the $\mathrm{P}_{(\mathrm{a})} \mathrm{O}_{2}$ to hyperoxic levels resulted in vasoconstriction in $91 \%$ of the studies. In $7 \%$ there 
Table 1 In vivo studies

\begin{tabular}{|c|c|c|c|c|c|c|c|}
\hline 1st Author, year of publication & Effect* & Species (strain) & Organ/tissue & Diameter $(\mu \mathrm{m})$ & Vessels (n) & Oxyge & \\
\hline Duling, 1974 [38] & $\downarrow$ & Hamster (GS) & Cheek pouch muscle & 15 & 14 & $0 /$ & $21 \%$ \\
\hline Gorczynski \& Duling, 1978 \# [61] & $\downarrow$ & Hamster & Cremaster muscle & 13 & 3 & $0 /$ & $10 \%$ \\
\hline Davis et al., 1981 a [85] & $\downarrow$ & Hamster (GS) & Cheek pouch muscle & 26 & 6 & $0 /$ & $20 \%$ \\
\hline Davis et al., 1981 b [85] & $\downarrow$ & Hamster (GS) & Cheek pouch muscle & 14 & 6 & $0 /$ & $20 \%$ \\
\hline Davis et al., 1981 c [85] & $\downarrow$ & Hamster (GS) & Cheek pouch muscle & 96 & 6 & $0 /$ & $20 \%$ \\
\hline Davis et al., 1981 d [85] & $\downarrow$ & Hamster (GS) & Cheek pouch muscle & 51 & 6 & $0 /$ & $20 \%$ \\
\hline Lombard et al., 1981 a [86] & $\downarrow$ & Hamster & Cheek pouch muscle & 19 & 10 & $0 /$ & $10 \%$ \\
\hline Lombard et al., 1981 b [86] & $\downarrow$ & Hamster & Cheek pouch muscle & 9 & 7 & $0 /$ & $10 \%$ \\
\hline Sullivan \& Johnson, 1981 a [36] & $\downarrow$ & Cat & Sartorius muscle & 52 & 11 & $0 /$ & $20 \%$ \\
\hline Sullivan \& Johnson, 1981 b [36] & $\downarrow$ & Cat & Sartorius muscle & 35 & 16 & $0 /$ & $20 \%$ \\
\hline Sullivan \& Johnson, 1981 c [36] & $\downarrow$ & Cat & Sartorius muscle & 18 & 10 & $0 /$ & $20 \%$ \\
\hline Sullivan \& Johnson, 1981 d [36] & $\downarrow$ & Cat & Sartorius muscle & 13 & 6 & $0 /$ & $20 \%$ \\
\hline Sullivan \& Johnson, 1981 e [36] & $\downarrow$ & Cat & Sartorius muscle & 9 & 10 & $0 /$ & $20 \%$ \\
\hline Sullivan \& Johnson, 1981 f [36] & $\downarrow$ & Cat & Sartorius muscle & 8 & 12 & $0 /$ & $20 \%$ \\
\hline Jackson \& Duling, 1983 [59] & $\downarrow$ & Hamster (GS) & Cheek pouch muscle & 32 & 19 & $0 /$ & $21 \%$ \\
\hline Lombard \& Stekiel, 1985 a [57] & $\downarrow$ & Rat (WS) & Mesoappendix & 41 & 16 & $0 /$ & $21 \%$ \\
\hline Lombard \& Stekiel, 1985 b [57] & $\downarrow$ & Rat (WS) & Mesoappendix & 19 & 11 & $0 /$ & $21 \%$ \\
\hline Lombard \& Stekiel, 1985 c [57] & $\leftrightarrow$ & Rat (WS) & Mesoappendix & 11 & 15 & $0 /$ & $21 \%$ \\
\hline Jackson, 1986 a [32] & $\downarrow$ & Hamster (GS) & Cheek pouch muscle & 20 & 19 & $0 /$ & $21 \%$ \\
\hline Jackson, 1986 b [32] & $\downarrow$ & Hamster (GS) & Cremaster muscle & 14 & 6 & $0 /$ & $21 \%$ \\
\hline Jackson, 1986 c [32] & $\downarrow$ & Rat (SD) & Cremaster muscle & 19 & 8 & $0 /$ & $21 \%$ \\
\hline Jackson, 1987 [12] & $\downarrow$ & Hamster (GS) & Cheek pouch muscle & 40 & 5 & $0 /$ & $21 \%$ \\
\hline Jackson, 1988 [62] & $\downarrow$ & Hamster (GS) & Cheek pouch muscle & 31 & 22 & $0 /$ & $21 \%$ \\
\hline Lombard \& Stekiel, 1988 a [66] & $\downarrow$ & Rat (WKY) & Mesoappendix & 30 & 10 & $0 /$ & $10 \%$ \\
\hline Lombard \& Stekiel, 1988 b [66] & $\downarrow$ & Rat (WKY) & Mesoappendix & 18 & 15 & $0 /$ & $10 \%$ \\
\hline Lombard \& Stekiel, 1988 c [66] & $\downarrow$ & Rat (WKY) & Mesoappendix & 10 & 11 & $0 /$ & $10 \%$ \\
\hline Jackson, 1989 [63] & $\downarrow$ & Hamster (GS) & Cheek pouch muscle & 28 & 19 & $0 /$ & $21 \%$ \\
\hline Bertuglia et al., 1991 a [43] & $\downarrow$ & Hamster (GS) & Dorsal skin & 5 & 20 & $.21 /$ & 1.0 \\
\hline Bertuglia et al., 1991 b [43] & $\downarrow$ & Hamster (GS) & Dorsal skin & 7 & 12 & $.21 /$ & 1.0 \\
\hline Bertuglia et al., 1991 c [43] & $\uparrow$ & Hamster (GS) & Dorsal skin & 46 & 10 & $.21 /$ & 1.0 \\
\hline Jackson, 1991 [67] & $\downarrow$ & Hamster (GS) & Cheek pouch muscle & 12 & 11 & $0 /$ & $21 \%$ \\
\hline Taguchi et al., 1992 [87] & $\downarrow$ & Rabbit & Ear & $?$ & 6 & $.21 /$ & 1.0 \\
\hline Jackson, 1993 a [64] & $\downarrow$ & Hamster (GS) & Cremaster muscle & 16 & 22 & $0 /$ & $21 \%$ \\
\hline Jackson, 1993 b [64] & $\downarrow$ & Hamster (GS) & Cheek pouch muscle & 20 & 15 & $0 /$ & $21 \%$ \\
\hline Rafi \& Boegehold, 1993 a [33] & $\downarrow$ & Rat (SS/Jr) & Spinotrapezius & 29 & 13 & $0 /$ & $10 \%$ \\
\hline Rafi \& Boegehold, 1993 b [33] & $\downarrow$ & Rat (SS/Jr) & Spinotrapezius & 17 & 17 & $0 /$ & $10 \%$ \\
\hline Rafi \& Boegehold, 1993 c [33] & $\downarrow$ & Rat (SS/Jr) & Spinotrapezius & 9 & 16 & $0 /$ & $10 \%$ \\
\hline Pries et al., 1995 [88] & $\downarrow$ & Rat (SD) & Spinotrapezius & 35 & 18 & $0 /$ & $20 \%$ \\
\hline Dewhirst et al., 1996 [58] & $\leftrightarrow$ & Rat (F344) & Dorsal skin & 47 & 6 & $.21 /$ & 1.0 \\
\hline Harder et al., 1996 [89] & $\downarrow$ & Rat (SD/WS) & Cremaster muscle & 21 & 8 & $0 /$ & $5 \%$ \\
\hline Welsh et al., 1998 [60] & $\downarrow$ & Hamster (GS) & Cheek pouch muscle & 52 & 17 & $0 /$ & $21 \%$ \\
\hline Lombard et al., 1999 a [90] & $\downarrow$ & Hamster (GS) & Cremaster muscle & 19 & 19 & $0 /$ & $21 \%$ \\
\hline Lombard et al., 1999 b [90] & $\downarrow$ & Hamster (GS) & Retractor & 22 & 11 & $0 /$ & $21 \%$ \\
\hline Lombard et al., 1999 c [90] & $\downarrow$ & Hamster (GS) & Cheek pouch muscle & 21 & 10 & $0 /$ & $21 \%$ \\
\hline
\end{tabular}


Table 1 In vivo studies (Continued)

\begin{tabular}{|c|c|c|c|c|c|c|c|}
\hline 1st Author, year of publication & Effect* $^{*}$ & Species (strain) & Organ/tissue & Diameter $(\mu \mathrm{m})$ & Vessels (n) & Oxyg & \\
\hline Frisbee \& Lombard, 1999 [69] & $\downarrow$ & Rat (SD) & Cremaster muscle & 20 & 12 & $0 /$ & $21 \%$ \\
\hline Frisbee \& Lombard, 2000 [91] & $\downarrow$ & Rat (SD) & Cremaster muscle & 17 & 12 & $0 /$ & $21 \%$ \\
\hline Frisbee et al., 2000 [92] & $\downarrow$ & Rat (WKY) & Cremaster muscle & 18 & 6 & $0 /$ & $21 \%$ \\
\hline Sauls \& Boegehold, 2000 [34] & $\leftrightarrow$ & Rat (SD) & Ileum & 63 & 11 & $5 /$ & $21 \%$ \\
\hline Komori et al., 2001 [68] & $\downarrow$ & Rabbit (Albino) & Ear & $\mathrm{nr}$ & 11 & $.21 /$ & 1.0 \\
\hline Kunert et al., 2001 a [73] & $\downarrow$ & Rat (SD) & Cremaster muscle & $\mathrm{nr}$ & 16 & $0 /$ & $21 \%$ \\
\hline Kunert et al., 2001 b [72] & $\downarrow$ & Rat (WKY) & Cremaster muscle & $\mathrm{nr}$ & 26 & $0 /$ & $21 \%$ \\
\hline Sauls \& Boegehold, 2001 [35] & $\leftrightarrow$ & Rat (SD) & Ileum & 62 & 17 & $5 /$ & $21 \%$ \\
\hline Frisbee, 2002 [11] & $\downarrow$ & Rat (SD) & Cremaster muscle & 22 & 12 & $0 /$ & $21 \%$ \\
\hline Frisbee \& Lombard, 2002 [31] & $\downarrow$ & Rat & Cremaster muscle & 102 & 12 & $0 /$ & $21 \%$ \\
\hline Tsai et al., 2003 a [45] & $\downarrow$ & Hamster (GS) & Dorsal skin & 59 & 18 & $.21 /$ & 1.0 \\
\hline Tsai et al., 2003 b [45] & $\downarrow$ & Hamster (GS) & Dorsal skin & 25 & 15 & $.21 /$ & 1.0 \\
\hline Tsai et al., 2003 c [45] & $\downarrow$ & Hamster (GS) & Dorsal skin & 10 & 15 & $.21 /$ & 1.0 \\
\hline Tsai et al., 2003 d [45] & $\downarrow$ & Hamster (GS) & Dorsal skin & 6 & 15 & $.21 /$ & 1.0 \\
\hline Drenjancevic et al., 2004 a [65] & $\downarrow$ & Rat (RGRR) & Cremaster muscle & 19 & 8 & $5 /$ & $21 \%$ \\
\hline Drenjancevic et al., 2004 b [65] & $\downarrow$ & Rat (Dahl) & Cremaster muscle & 22 & 8 & $5 /$ & $21 \%$ \\
\hline Cabrales et al., 2006 [44] & $\downarrow$ & Hamster (GS) & Dorsal skin & 58 & 26 & $.21 /$ & 1.0 \\
\hline Sakai et al., 2007 [37] & $\downarrow$ & Rat (WS) & Sciatic nerve & 26 & 8 & $0 /$ & $21 \%$ \\
\hline Kunert et al., 2009 [93] & $\downarrow$ & Rat (SD) & Cremaster muscle & 23 & 18 & $0 /$ & $21 \%$ \\
\hline Wang et al., 2009 [94] & $\downarrow$ & Rat (Dahl SS) & Cremaster muscle & 16 & 9 & $0 /$ & $21 \%$ \\
\hline Ngo et al., 2010 [41] & $\downarrow$ & Mouse (C57) & Cremaster muscle & 29 & 6 & $0 /$ & $21 \%$ \\
\hline Riemann et al., 2010 [42] & $\downarrow$ & Mouse (C57) & Cremaster muscle & 31 & 7 & $0 /$ & $95 \%$ \\
\hline Messmer et al., 2012 [14]キ & $\leftrightarrow$ & Hamster (GS) & Dorsal skin & $\mathrm{nr}$ & $?$ & $.21 /$ & 1.0 \\
\hline Ngo et al., 2013 [40] & $\downarrow$ & Mouse (C57) & Cremaster muscle & 33 & 28 & $0 /$ & $21 \%$ \\
\hline Milstein et al., 2016 [39] & $\downarrow$ & Rabbit (NZW) & Sublingual & 4 & 40 & $.21 /$ & 1.0 \\
\hline
\end{tabular}

Studies are sorted based on year of publication

Abbreviations: NZW New Zealand White, SD Sprague Dawley, WS Wistar, $L Y$ Landrace Yorkshire, $M$ muscle chamber, $W$ wire myograph, $P$ pressure myograph, $n r$ not reported

*Effect as reported by the original paper, arrows indicate the direction of the change in diameter

**A value suffixed with a "\%" symbol indicates the percentage of oxygen used to oxygenate the physiological salt solution; other values indicate the fraction of inspired oxygen

\#Not included in the meta-analysis due to a small number of observations or because no data was shown ( $\neq$ )

was no effect on arteriolar diameter $[35,57,58]$ and in one study dilation of second order arterioles was observed [43]. The median diameter change was $-20 \%$ with a range of $-64 \%$ to $+6 \%$.

\section{Ex vivo effect of hyperoxia on vascular tone}

Including the experiments without data, hyperoxic vasoconstriction was seen in $59 \%$ of the reports. In $23 \%$ there was no change in arteriolar diameter $[15,16,51,53]$ and in $18 \%$ dilation was observed $[15,17,18,52]$. In the studies that reported diameters $[13,16,47-50]$, the median change was $-14 \%$ with a range of $-24 \%$ to $-1 \%$.

\section{Experimental difficulties}

Some articles explicitly mentioned difficulties encountered during the experiments and variation in responses between separate vessels. In vivo, a vasomotor response to changes in oxygen exposure was not observed in $12 \%$ [38], 25\% [59] and 40\% [42] of the animals. The authors did not provide an explanation for non-responsiveness in these animals. In two studies, $26-50 \%$ of the vessels failed to return to their stable baseline diameter after the first exposure to hyperoxia [41, 60].

Ex vivo, one study described that only $32 \%$ of the isolated arteries responded to oxygen [59]. In addition, in reactive vessels, the constriction to oxygen disappeared after 2-6 oxygen exposure cycles. The author concluded that the lack of oxygen reactivity was not an indication of vessel damage, because both responding and non-responding vessels developed spontaneous tone and had similar responses to norepinephrine. In porcine coronary arteries, repeated exposures to 
Table 2 Ex vivo studies

\begin{tabular}{|c|c|c|c|c|c|c|c|c|c|c|}
\hline 1st Author & Effect $^{*}$ & Species (strain) & Artery & Type & Tone & Model & Diameter $(\mu \mathrm{m})$ & Vessels (n) & Oxygen* & \\
\hline Chang \& Detar, 1980 [54] & $\downarrow$ & Rabbit (NZW) & Aorta & Conduit & Norepinephrine & M & $4000-5000$ & 10 & $100 /$ & 300 \\
\hline $\begin{array}{l}\text { Pittman \& Graham, } \\
1986 \text { a [55]‡ }\end{array}$ & $\downarrow$ & Rabbit (NZW) & Aorta & Conduit & Norepinephrine & M & $\mathrm{nr}$ & $?$ & $\pm 100 /$ & 280 \\
\hline $\begin{array}{l}\text { Pittman \& Graham, } \\
1986 \text { b [55]‡ }\end{array}$ & $\downarrow$ & Rabbit (NZW) & Aorta & Conduit & $\mathrm{KCl}$ & M & $\mathrm{nr}$ & $?$ & $\pm 100 /$ & 280 \\
\hline Vallet et al., 1994 [56] & $\downarrow$ & Rat (SD) & Aorta & Conduit & Phenylephrine & M & $2200-2800$ & 6 & $20 /$ & 95 \\
\hline Day et al., 1992 [51] & $\leftrightarrow$ & Rabbit (NZW) & Aorta & Conduit & None & W & $\mathrm{nr}$ & 6 & $160 /$ & 495 \\
\hline Kwan et al., 1989 [52] & $\uparrow$ & Sheep & Coronary & Conduit & None & M & $600-2500$ & 8 & $12 /$ & 95 \\
\hline Ngai et al., 1990 [17] & $\uparrow$ & Pig & Coronary & Conduit & None & M & $\mathrm{nr}$ & 10 & $20 /$ & 95 \\
\hline Rubanyi \& Paul, 1984 b [15] & $\uparrow$ & Pig & Coronary & Conduit & Histamine & M & $\mathrm{nr}$ & 6 & $40 /$ & 95 \\
\hline Rubanyi \& Paul, 1985 [18] & $\uparrow$ & Pig & Coronary & Conduit & Spontaneous/KCl & M & $1500-2000$ & 10 & $12 /$ & 95 \\
\hline Hedegaard et al., 2011 [70] & $\downarrow$ & Pig (LY) & Coronary & Conduit & PGF2a & W & $\mathrm{nr}$ & 9 & $10 /$ & 950 \\
\hline Kalsner, 1976 [46] & $\downarrow$ & Cow & Coronary & Conduit & Spontaneous & M & $\mathrm{nr}$ & 14 & $112 /$ & 515 \\
\hline Pasgaard et al., 2007 b [53] & $\downarrow$ & Pig & Coronary & Conduit & U46199 & W & $\mathrm{nr}$ & 14 & $120 /$ & 617 \\
\hline Pasgaard et al., 2007 c [53] & $\downarrow$ & Pig & Coronary & Conduit & $5-\mathrm{HT}$ & W & $\mathrm{nr}$ & 7 & $120 /$ & 617 \\
\hline Pasgaard et al., 2007 a [53] & $\leftrightarrow$ & Pig & Coronary & Conduit & None & W & $\mathrm{nr}$ & $?$ & $120 /$ & 617 \\
\hline Rubanyi \& Paul, 1984 a [15]‡ & $\leftrightarrow$ & Cow & Coronary & Conduit & $\mathrm{KCl}$ & M & $\mathrm{nr}$ & $?$ & $40 /$ & 95 \\
\hline Frisbee \& Lombard, 2002 [31]\# & $\downarrow$ & Rat & Cremaster & Resistance & Spontaneous & $P$ & 140 & 12 & $10 /$ & 2 \\
\hline Messina et al., 1994 [13] & $\downarrow$ & Rat (WS) & Cremaster & Resistance & Spontaneous & $P$ & 163 & 27 & $21 /$ & 95 \\
\hline Smit et al., 2017 b [16] & $\leftrightarrow$ & Mouse (C57) & Femoral & Conduit & Norepinephrine & $P$ & 293 & 16 & $78 /$ & 375 \\
\hline Fredricks et al., 1994 [47] & $\downarrow$ & Rat (SD) & Gracilis & Resistance & Spontaneous & $P$ & $100-300$ & 7 & $87 /$ & 148 \\
\hline Frisbee et al., 2001 [48] & $\downarrow$ & Rat (SD) & Gracilis & Resistance & Spontaneous & $P$ & 185 & 15 & $15 /$ & 95 \\
\hline Frisbee et al., 2002 [49] & $\downarrow$ & Rat (SD) & Gracilis & Resistance & Spontaneous & $P$ & 194 & 76 & $10 /$ & 2 \\
\hline Liu et al., 1997 [50] & $\downarrow$ & Rat (SD) & Gracilis & Resistance & Spontaneous & $P$ & $\mathrm{nr}$ & 6 & $10 /$ & $21 \%$ \\
\hline Smit et al., 2017 a [16] & $\leftrightarrow$ & Mouse (C57) & Gracilis & Resistance & Spontaneous & $P$ & 129 & 22 & $78 /$ & 375 \\
\hline
\end{tabular}

Studies are sorted based on artery, effect and first author

Abbreviations: NZW New Zealand White, SD Sprague Dawley, WS Wistar, LY Landrace Yorkshire, $M$ muscle chamber, $W$ wire myograph, $P$ pressure myograph, $n r$ not reported

*Effect as reported by the original paper; arrows indicate the direction of the effective change in diameter

${ }^{* *} \mathrm{~A}$ value suffixed with a "\%" symbol indicates the percentage of oxygen used to oxygenate the physiological salt solution, other values indicate the oxygen tension in $\mathrm{mmHg}$

¥Not included in the meta-analysis because no data was shown or values could not be recalculated (\#). For diameters, values are presented as range or average diameter

hyperoxia also decreased the magnitude of hyperoxic vasoconstriction.

\section{Dose-response studies}

Dose-response relationships were examined in 16 in vivo studies: 10 of these used three oxygen concentrations [39, 41, 57, 61-67] (including reference level), in four studies four levels of oxygen were used $[59,68,69]$ and in two studies five different oxygen concentrations were used [11, $31]$. In the majority of these studies, $20-21 \%$ oxygen in the superfusion buffer was the maximal exposure, while one study also used $95 \%$ oxygen [59]. Intermediate oxygen concentrations were $5 \%, 7 \%$ or $10 \%$ oxygen. In studies with more than three different oxygen doses, the relationship between diameter changes and oxygen concentration was approximated as sigmoidal [11], exponential [59, 68] or linear $[31,69]$. In general, arteriolar diameter decreased as the oxygen concentration increased, without any apparent threshold dose. Only in one study, in which the relationship seemed sigmoidal [11], no constriction occured until the oxygen concentration in the superfusion buffer was increased above $5 \%$.

Of the ex vivo studies, eight studies used a total of three $[18,31,47,49]$, four $[16,50,70]$ or five [55] oxygen concentrations (including reference level). The range of oxygen exposure assessed varied between 10 and $21 \%$ to $10-95 \%$. In all studies except for two, a decrease in diameter was seen as the oxygen concentration increased. The dose-response relationships in studies with more than three levels of exposure were described as linear in the normoxic-hyperoxic range [16, 55, 70, 71]. In one study there was no discernable difference between $40 \%$ or $95 \% \mathrm{O}_{2}$ [18] and in another there was no effect at any oxygen concentration $\left(10-95 \% \mathrm{O}_{2}\right)$ [16]. 


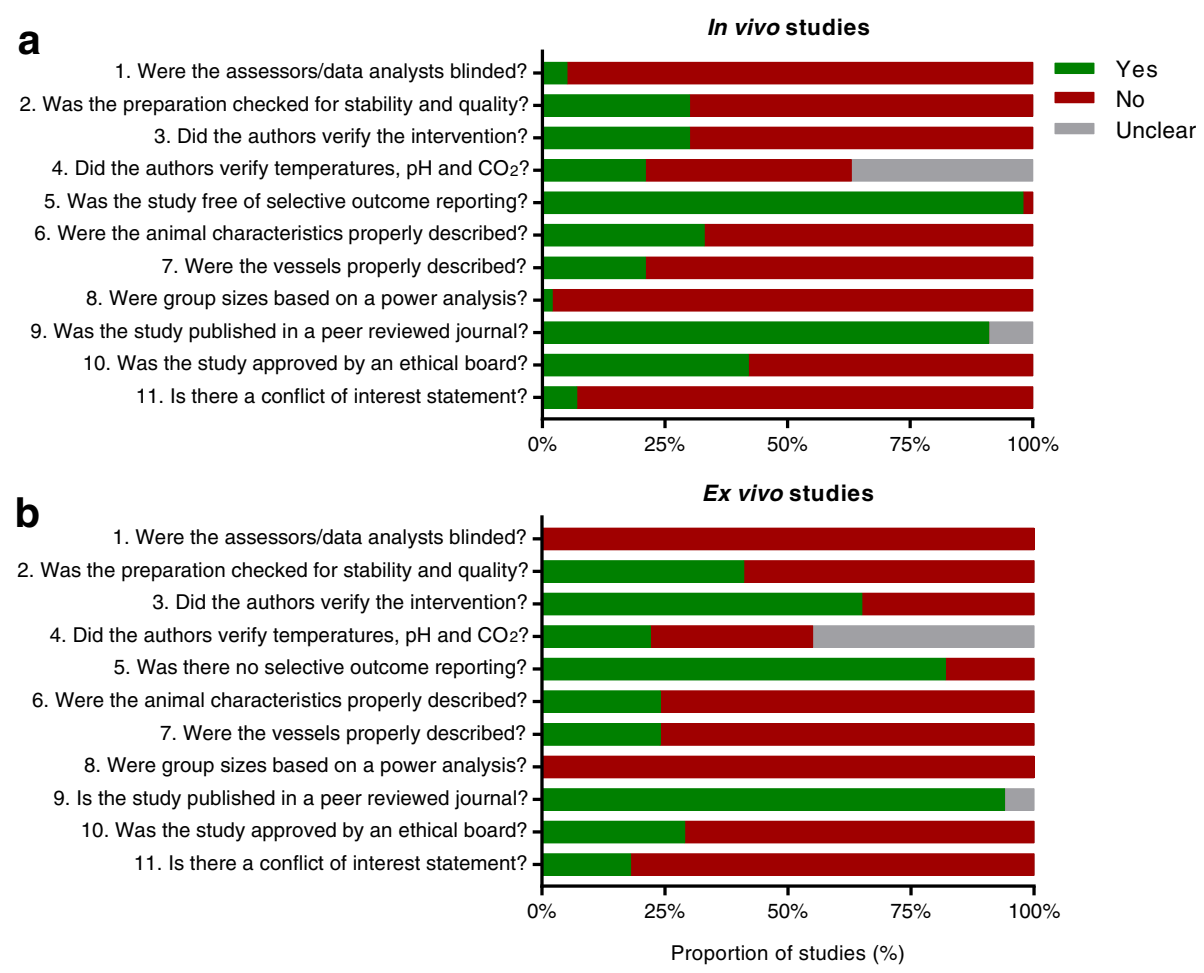

Fig. 2 Quality assessment scores for in vivo studies (a) and ex vivo studies (b)

\section{Meta-analysis}

\section{In vivo studies}

Out of the 69 experiments, $67(\mathrm{k}=67)$ were included in the meta-analysis of in vivo studies. Two could not be included because in one study only three vessels were observed [61] and in another the data were not shown [14]. Overall, there was a significant vasoconstrictor effect of hyperoxia on arteries in vivo (SMD - $1.42(-1.65$ to -1.19$)$, Fig. 3); heterogeneity between studies was high $\left(I^{2} 72 \%\right.$ [62-85]).

\section{Ex vivo studies}

Out of the 23 experiments, $18(\mathrm{k}=18)$ were included in the meta-analysis of ex vivo studies. Four experiments were mentioned in the text but were not accompanied by data $[15,53,55]$ and one paper reported normalized data for one experiment that could not be appropriately recalculated [31]. Overall, there was a borderline significant vasoconstrictor effect of hyperoxia on arteries ex vivo (SMD -0.56 ( -1.09 to -0.03$), P=0.04$, Fig. 4). Between-study heterogeneity was high $\left(I^{2} 88 \%\right.$ [80-87]).

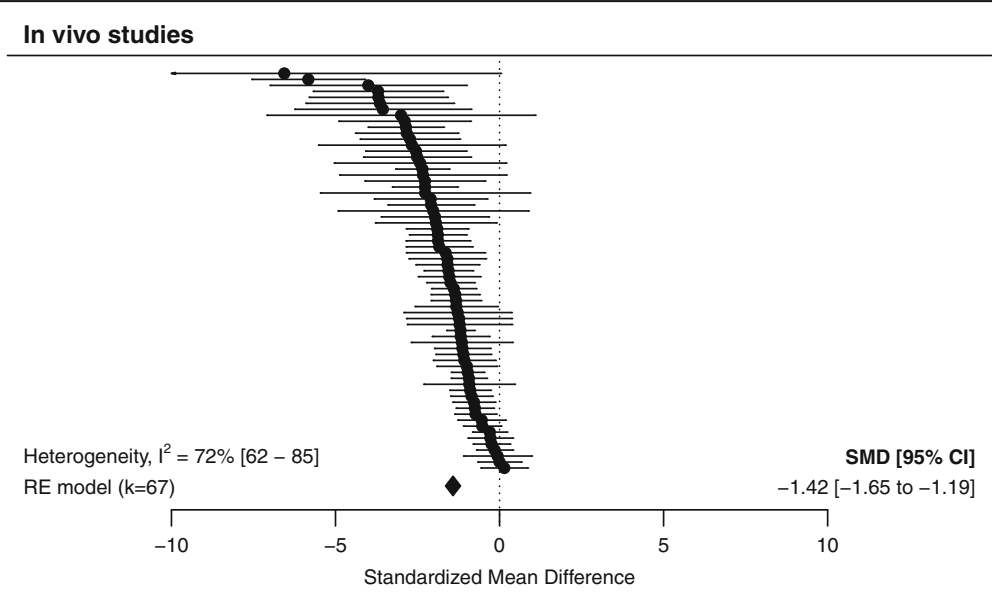

Fig. 3 In vivo studies on the effect of hyperoxia on arteriolar diameter, sorted by effect size 


\section{Ex vivo studies

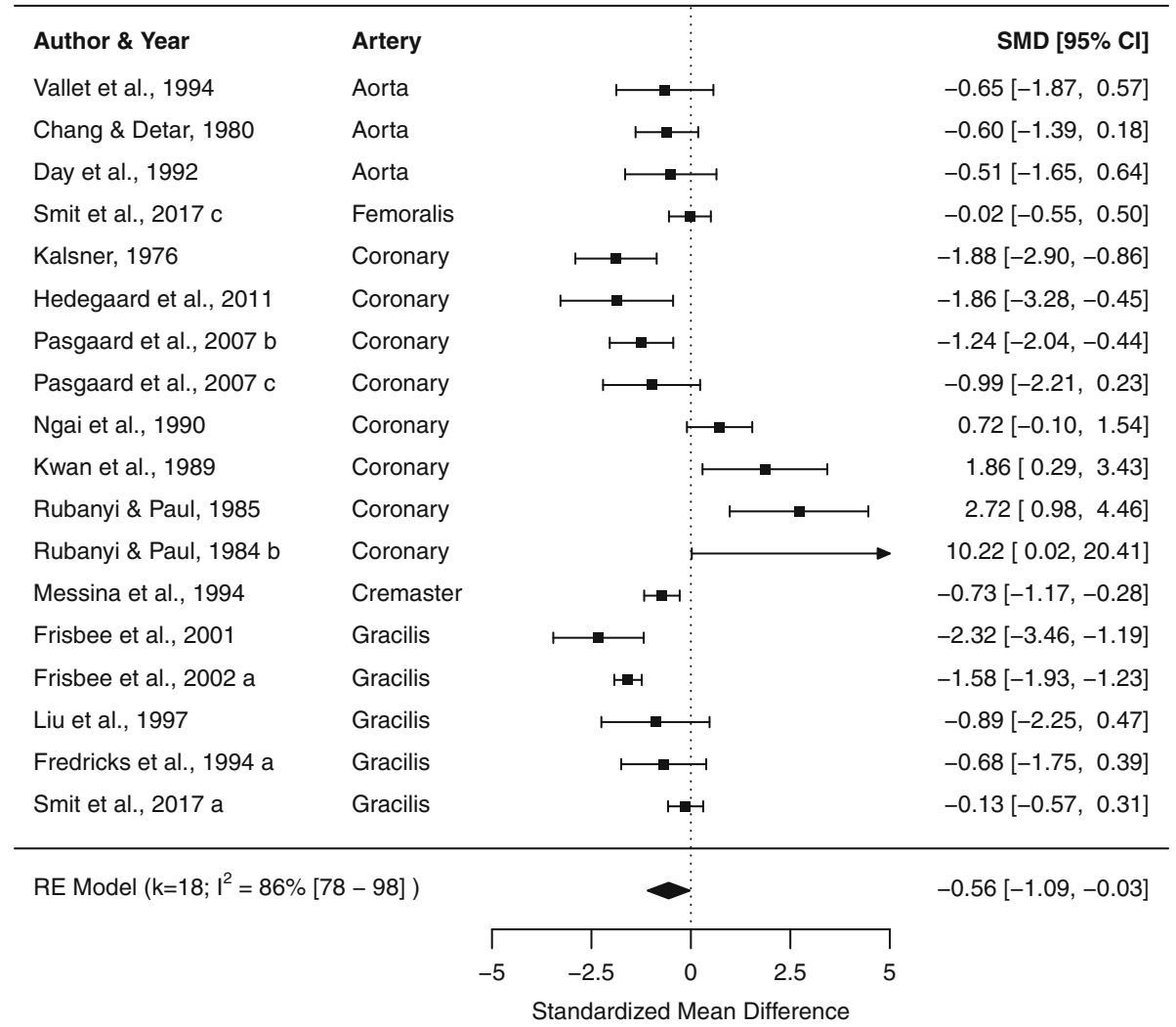

Fig. 4 Data sets from ex vivo studies on vascular diameter or tone. The plot is sorted by artery type and effect size. SMD, standardized mean difference

\section{Exploring sources of heterogeneity In vivo studies}

Subgroup analyses were only conducted in groups with five or more experiments from at least three unique studies. For the in vivo studies, subgroup analyses were conducted for species (rat and hamster, Fig. 5), vascular region (dorsal skin, intestines, cremaster muscle, cheek pouch, Fig. 5), method of hyperoxia induction (systemic by increasing the percentage of oxygen in the inspired air, Fig. 5) and vessel diameter (Fig. 6). Sex could not be examined because the studies mostly used male animals or did not specifically report animal sex. Species did not modify the overall effect $(P=0.83)$. Vascular region interacted with the overall effect $(P<0.001)$. $P$ values for further pairwise comparisons were adjusted with the HolmsBonferroni method. Moderators that increased the effect size of hyperoxic constriction were the cremaster as a vascular region $\left(P_{\text {holm }}<0.001\right)$. This subgroup explained 53\% of the heterogeneity $(R[2])$ in the overall effect. A smaller response was seen in studies in which the dorsal skin $\left(P_{\text {holm }}=0.033, R^{2}=10 \%\right)$ or the intestines was investigated (mesoappendix and ileum, $P_{\text {holm }}=0.033, R^{2}=19 \%$ ). The effect size was not different in studies that induced hyperoxia systemically $\left(P_{\text {holm }}=0.061, R^{2}=7 \%\right)$. In a weighted four-knot restricted cubic spline meta-regression, artery diameter accounted for $16 \%$ of the observed heterogeneity $(P=0.008$, Fig. 6).

\section{Ex vivo studies}

In the ex vivo studies, only two species were used in a sufficient number of studies to be considered as subgroups (pigs and rats). Other potential sources of heterogeneity investigated were type of artery (conduit or resistance arteries) and vessel origin (gracilis and coronary arteries). Coincidentally, all conduit arteries were examined in tension myographs, while resistance arteries were mounted in pressure myographs. Hence, results of an analysis based on the method used to mount the isolated arteries would be the same as now presented based on the type of artery. There were not sufficient studies reporting sex or using flow to analyze as subgroups. Of the investigated subgroups, none explained a significant proportion of the heterogeneity (Fig. 7).

\section{Publication bias and sensitivity analysis}

The funnel plot for the in vivo studies shows an indication of publication bias, favoring studies that reported 


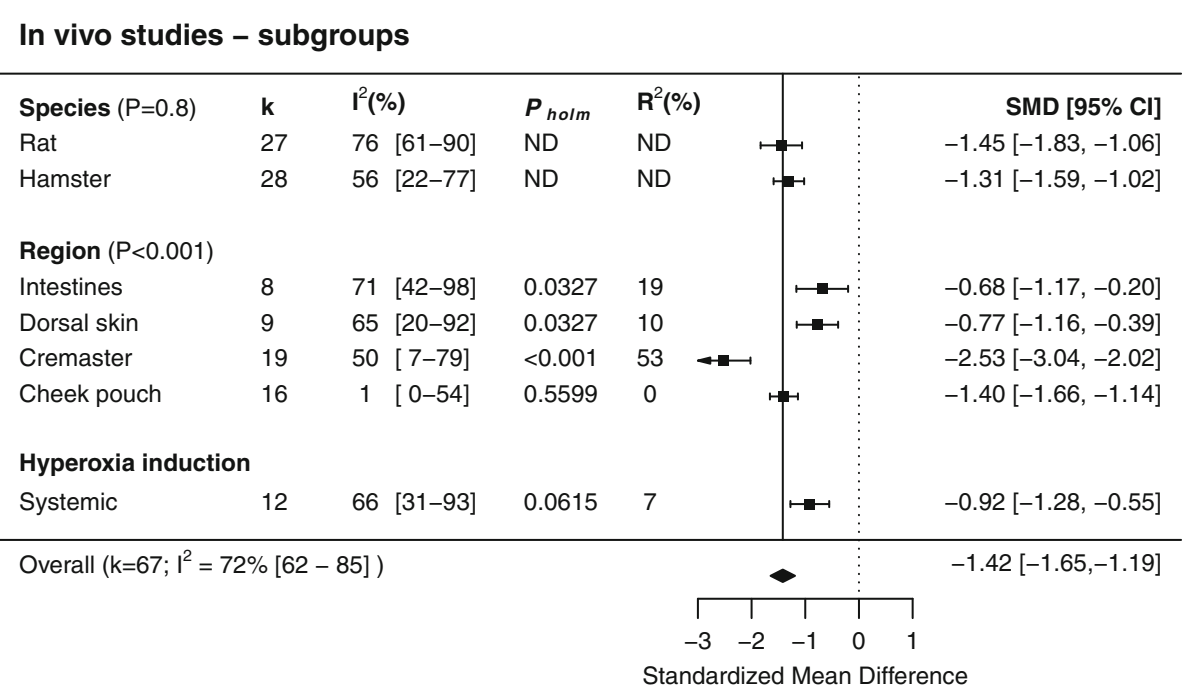

Fig. 5 Subgroup analyses of in vivo studies. Hyperoxia induced more constriction in the cremaster vasculature. Hyperoxic vasoconstriction was reduced in the dorsal skin and intestines (mesoappendix and ileum). $P_{\text {holm }}$ values are corrected for multiple testing with the Holm-Bonferroni method. ND, not determined; $k$, data sets

constriction of arterioles upon exposure to hyperoxia (Additional file 3). Balancing the results with the trim and fill method reduced the overall effect size $(P=0.03)$ from $-1.42(-1.65$ to -1.19$)$ to $-1.03(-1.30$ to -0.76$)$. The funnel plot for the ex vivo studies did not reveal evidence of publication bias (Additional file 3).

For all analyses shown, we used a conservative correlation coefficient of 0.7 to impute missing standard deviations of paired data. Missing standard deviations had to be calculated for $21 / 67$ in vivo data sets and 7/18 ex vivo data sets. Using a higher or lower correlation coefficient did not affect the results for the in vivo analysis (Additional file 4). For the ex vivo analysis, the differences were marginal, but when a low correlation coefficient of 0.5 was used, the confidence interval of the overall effect crossed zero.

\section{Discussion}

In this systematic review and meta-analysis, we found that normobaric hyperoxia or hyperoxic superfusion decreases the diameter of in vivo arteries and arterioles, in comparison to normoxia or normoxic superfusion, indicating an increase in vascular tone. For ex vivo studies, our meta-analysis shows a smaller effect of hyperoxia decrease in isolated vessel tone. Both vasodilation, vasoconstriction and neutral effects were observed, for which the explanation is unclear. In contrast, in the intact animal hyperoxia almost exclusively causes vasoconstriction

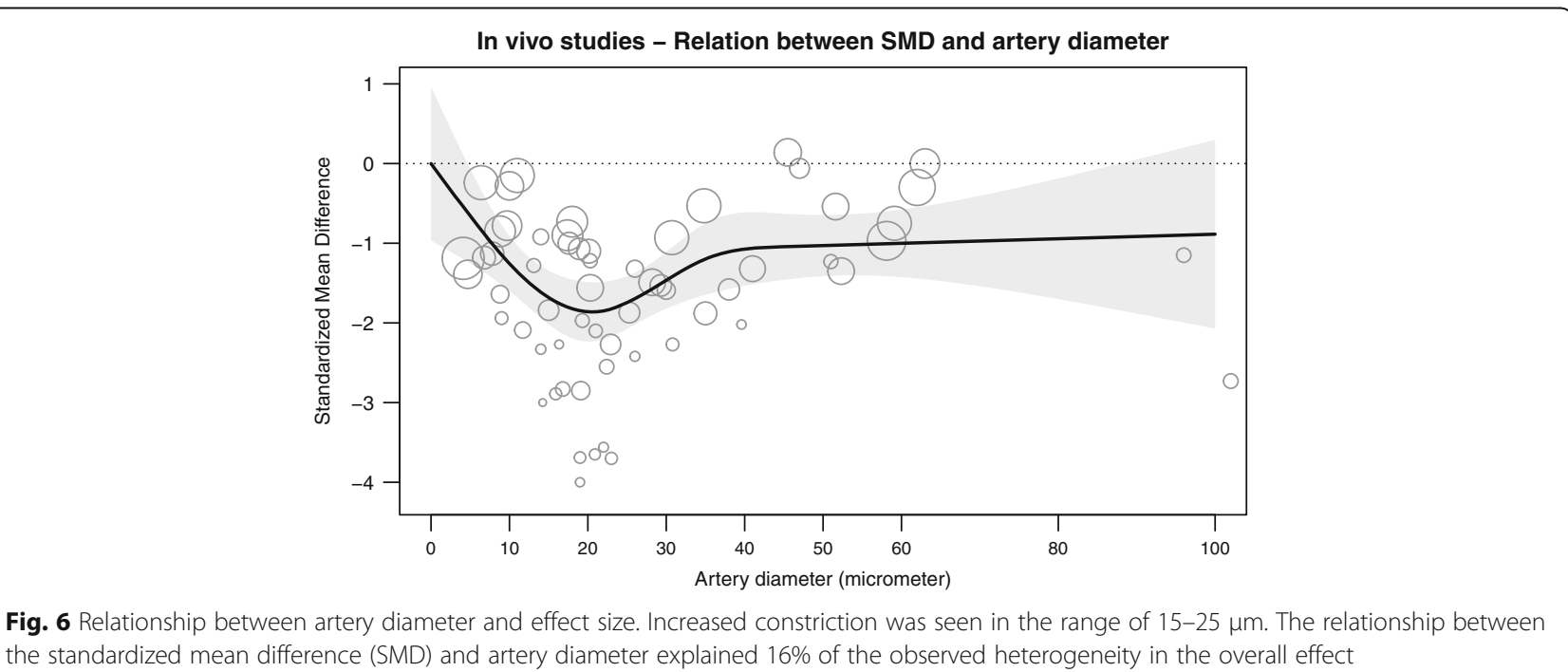




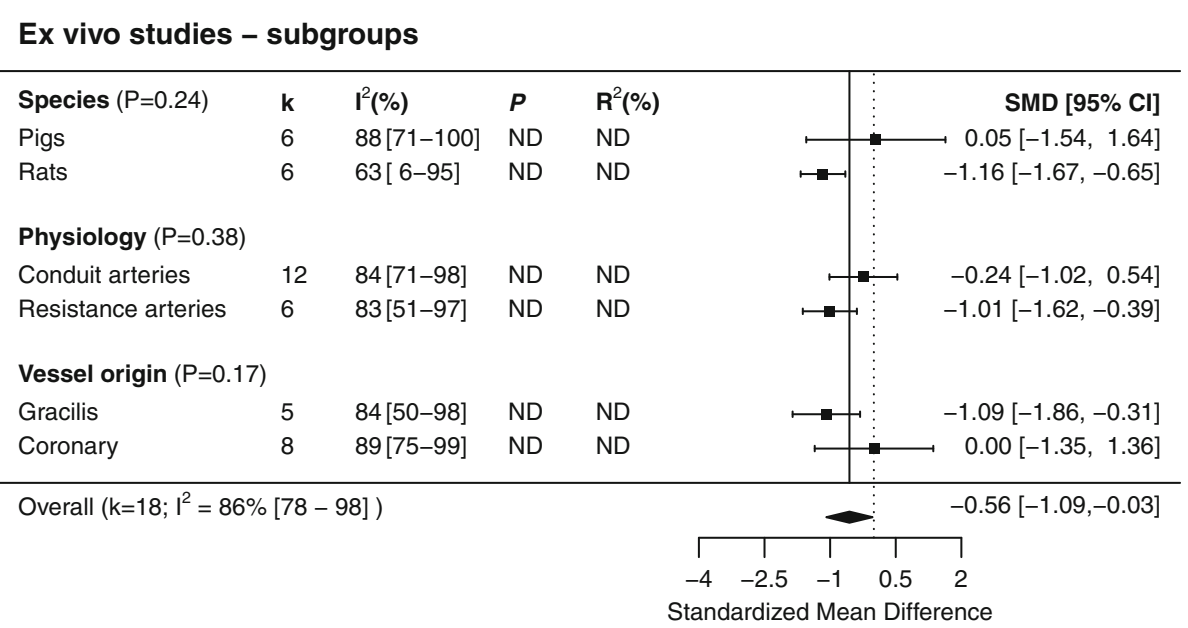

Fig. 7 Subgroup analyses for ex vivo studies. None of the investigated subgroups explained a significant proportion of the heterogeneity. $K$, data sets; SMD, standardized mean difference

(median $-20 \%$ reduction in diameter), which was most pronounced in the cremaster vasculature but less in the intestinal and skin vasculature. In a post-hoc analysis, the magnitude of hyperoxic vasoconstriction showed a U-shaped curve and was highest in vessels of $15-25 \mu \mathrm{m}$ in diameter. There seems to be no oxygen threshold for the constriction to occur.

There was a large difference in effect size between the in vivo and ex vivo studies. The existence of a branching order-specific effect, i.e. confinement of hyperoxic vasoconstriction to smaller arterioles, could be one explanation for this difference. We found that oxygen-induced constriction was the largest in arteries of $15-25 \mu \mathrm{m}$ in diameter and less in arteries with diameters above and below this range. The arteries used in the ex vivo studies were all considerably larger than the vessels examined in vivo, ranging between $100 \mu \mathrm{m}$ and $5000 \mu \mathrm{m}$ ex vivo, versus 4-100 $\mu \mathrm{m}$ in vivo. The difference in size between the investigated arteries is the result of a practical limitation, considering that it is very difficult to isolate and mount small arteries without damaging the vessel ex vivo. The reduced effect of hyperoxia on arteries $<20 \mu \mathrm{m}$ may be explained by the decreasing presence of vascular smooth muscle and thus the ability to constrict. Another explanation is that the mechanism responsible for hyperoxic vasoconstriction is primarily located in extravascular tissue, rather than in the vessel wall. For example, the CYP450 omega hydroxylases, which can produce the vasoconstrictor 20-hydroxyeicosatetraenoic acid (20-HETE) from arachidonic acid, is found in cremaster arterioles, but is also highly expressed in the surrounding muscle tissue [72, 73]. Hence, for substantial (measurable) hyperoxic vasoconstriction, extravascular tissue may be required.

We found regional differences in the magnitude of hyperoxic constriction between the intestine, skin and cremaster muscle. The differences are likely related to intrinsic differences in blood vessels between vascular beds, or presence of multiple mechanisms that regulate vascular tone. For instance, 20-HETE constricts arteries in the cremaster and gracilis muscle but dilates mesenteric arteries [74]. Inhibition of nitric oxide in intestinal arteries only temporarily reduces vessel diameter [35], which suggests that in these vessels the impediment of one vasoactive system is quickly compensated by another. The same might be true for the pathway affected by hyperoxia. The reduced effect in skin could be related to the already low basal perfusion of skin at room temperature, so that any further reductions in flow and artery diameter are prevented by other mechanisms, such as temperature control [75]. In humans, hyperoxia has no effect on skin flow, unless first pharmacologically raised or measured in existing high-flow areas. Because control of body temperature is a major aspect of skin perfusion, the pathway for temperature control could take precedence over the oxygen sensor. The exact mechanism behind hyperoxic vasoconstriction has not yet been determined. Potential pathways include a reduction in nitric oxide bioavailability due to increased reactive oxygen species production, decreased production of prostaglandins, increased production of 20-HETE and interaction with calcium and potassium channels. The varying results may not necessarily be conflicting but could be simply due to differences in vascular beds and locations in the arterial tree.

This meta-analysis shows that hyperoxia in vivo causes a relatively increased level of vasoconstriction in muscle (i.e. cremaster and cheek pouch). Although perfusion of skeletal muscle is arguably not a priority in the critically ill, a restriction of blood flow in cardiac muscle may be detrimental. In one trial, hyperoxia led to increased 
infarct size in patients with myocardial infarction [76]; this may have been the result of decreased perfusion, considering that in pigs, hyperoxic coronary vasoconstriction leads to increased tissue hypoxia in areas with acute coronary stenosis [77]. The reduced constriction observed in the intestinal region could be of clinical importance. Organs with relatively reduced hyperoxic constriction will receive a larger portion of the cardiac output. This has been observed in hemodiluted dogs, pigs with peritonitis and rats with hemorrhagic shock, where hyperoxia caused a redistribution of the cardiac output towards vital organs such as the kidney, liver and intestines $[4,5,78]$. In rats, hyperoxia was shown to reduce bowel injury after ischemia and reperfusion of the mesentery [79]. Because of the relative increase in perfusion, hyperoxia may thus be beneficial for patients with splanchnic hypoperfusion.

Vessel diameter has a dominant influence on blood flow. In vivo, the median hyperoxia-induced decrease in diameter was $20 \%$, which, using Poiseuille's law, leads to a reduction of approximately $60 \%$ in flow. Following the dose-response studies, the constrictor effect of oxygen occurs at any level above normoxia, meaning that any level of hyperoxia will have consequences for organ blood flow if vasoconstriction occurs. However, the translatability of these effects to clinical practice is difficult. The effect of oxygen on artery diameter depends on oxygen tension, which was rarely measured in the included studies. The oxygen percentages used in superfusion buffers cannot be directly converted to a fraction of inspired oxygen. Second, in the majority of the in vivo studies, both the arteries and tissue were exposed to the hyperoxic oxygen tensions, meaning that the oxygen delivery was no longer solely facilitated by blood flow. Any tissue-derived signals generated by the reduction in blood flow may therefore have been missed, confounding the results.

Human hyperoxic coronary vasoconstriction is a well-documented phenomenon and has been observed directly by means of angiography [80, 81] and indirectly via increases in coronary vascular resistance measurements [80, 82-84]. Surprisingly however, results of ex vivo studies of the isolated coronary circulation are less univocal. Of the eight experiments with coronary vessels, vasoconstriction to increased oxygen tensions was seen in four, while vasodilation was observed in the other four. Differences could again be related to vessel size. In the studies in which coronary constriction was observed, arteries were mounted in wire myographs, which are suitable for vessels measuring $>100 \mu \mathrm{m}$. Unfortunately, these studies did not report vessel diameter. However, the studies in which dilation was seen used muscle chambers, which are generally used for large arteries. Two of these studies reported diameter, which ranged between 0.6 and $5 \mathrm{~mm}$. Another important consideration is that constriction was only seen with certain types of preconstriction. This was especially apparent in one study where coronary preparations were used without preconstriction or were constricted with serotonin (5HT) or thromboxane (U46619) analogues [53]. Hyperoxia had no effect on vessels without preconstriction, while a considerable hyperoxic constriction was seen in the precontracted vessels. Taken together; vessel preparation may be particularly important in ex vivo experiments and the use of isolated vessels may not be appropriate to study the complex vascular responses to hyperoxia.

\section{Limitations of this review and included studies}

There are several limitations to this meta-analysis. There was considerable heterogeneity between the in vivo studies, which could only in part be explained by differences in the region of interest and the size of the vessels investigated. For the ex vivo studies we did not find an explanation for the heterogeneity, suggesting the importance of a yet unidentified methodological, systemic or metabolic factor. Second, the funnel plots indicated publication bias for in vivo studies, favoring studies finding vasoconstriction in response to hyperoxia. However, correction with the trim and fill method did not significantly alter the conclusions. Third, due to the relatively small number of studies, some collinearity between the investigated subgroups may be present. Fourth, for some studies we had to make an assumption on the correlation coefficient to impute missing standard deviations. Our conservative estimate may have underestimated the effect, although our sensitivity analysis shows that using a higher correlation coefficient did not significantly alter the results. Fifth, all studies were performed with healthy animals; studies in disease models may give different results. Similarly, the use of live animals required the application of anesthetics in the majority of studies. Most of these drugs have at least some vasodilatory effects, which may have influenced the results. However, because of the pre-post design of the included studies, the effect of the anesthetic is anticipated to be the same under each oxygen condition. Sixth, we excluded studies on the lung, retinal and brain vasculature.

Finally, as reflected by our quality assessment of the included studies, the overall quality of the included studies was poor, which seriously hampers drawing reliable conclusions from the included animal studies. Baseline characteristics of animals were insufficiently described. Similarly, only 14 of the 60 studies properly described artery characteristics, such as vessel diameter and selection of vessels in the arterial tree. Reporting these data is important to increase reproducibility between studies. Similarly, measurement of $\mathrm{PO}_{2}, \quad \mathrm{pH}, \mathrm{PCO}_{2}$ and temperature was not (sufficiently) performed in the majority of studies. These measurements are crucial 
because of the dose response relationship between $\mathrm{PO}_{2}$ and hyperoxic vasoconstriction, and the effect of $\mathrm{pH}$, $\mathrm{PCO}_{2}$ and temperature on vascular tone. Without measuring $\mathrm{PO}_{2}$ the true intervention applied to the vasculature is unknown, which will contribute to heterogeneity. The lack of blinding in most studies may have led to an overestimation of the true effect. Another flaw in most studies is the absence of power calculations. Especially for studies aimed at discovering mechanisms responsible for oxygen-related changes in vessel diameter, a minimum predefined number of vessels is necessary to be able to draw objective statistical conclusions. The majority of the included studies were conducted before the institution of more rigorous legislation on the use of animals in scientific procedures known today. The overall poor quality of studies is therefore likely related to the absence of an ethical board reviewing the studies prior to their execution. Similarly, the same papers were published before editors put certain requirements on the reporting of study data. We have noted that the more recent papers were of considerably higher quality, which shows that the change in procedures concerning animal research, which are now becoming very similar to human trials in terms of ethical board review and data transparency, is for the better.

The majority of the in vivo studies were performed on thin, externalizable skeletal muscle. Studies in other critical visceral organs (e.g. liver, kidney) are currently lacking. We propose that future, high-quality studies focus on visceral organs such as the kidney and liver. These organs are highly relevant for patient care and the results will contribute to the rationale behind selective induction or avoidance of hyperoxia in certain types of patients.

\section{Conclusions}

In this meta-analysis of in vivo and ex vivo studies investigating the effect of hyperoxia on arteriolar diameter, we found a substantially larger effect size in vivo than ex vivo. The degree of the constriction is most pronounced in vessels of approximately $15-25 \mu \mathrm{m}$ in diameter and is proportional to the level of hyperoxia. The vasculature of the intestines and skin seem less sensitive to hyperoxic vasoconstriction than skeletal muscle. Ex vivo, the response of the coronary vasculature to hyperoxia is highly variable.

\section{Additional files}

Additional file 1: Search strategy. The search strategies used to search Pubmed and Embase for eligible studies. (PDF 201 kb)

Additional file 2: Quality checklist. Modified quality checklist used to assess the quality of the included studies. (PDF $14 \mathrm{~kb}$ )

Additional file 3: Funnel plots. Results of the publication bias assessment in the form of funnel plots. (PDF $344 \mathrm{~kb}$ )

Additional file 4: Sensitivity analysis. Results of the sensitivity analysis for the correlation coefficients. (PDF $232 \mathrm{~kb}$ )

\section{Abbreviations}

20 HETE: 20-Hydroxyeicosatetraenoic acid; AIC: Akaike information criteria; Cl: Confidence interval; e: Experiments; EDHF: Endothelium dependent hyperpolarizing factors; EDRF: Endothelium derived relaxing factors; FIO2: Fraction of inspired oxygen; $\mathrm{k}$ : Datasets; PaO2: Partial pressure of oxygen in arterial blood; PO2: Partial pressure of oxygen; SD: Standard deviation; SMD: Standardized mean difference; SYRCLE: The Systematic Review Centre for Laboratory Animal Experimentation

Funding

The authors received funding from ZonMW (project 114024107).

\section{Availability of data and materials}

The datasets used and/or analyzed during the current study are available from the corresponding author on reasonable request.

\section{Authors' contributions}

BS performed the database search, selected studies, extracted and analyzed the data and wrote the manuscript. YS helped with the interpretation of data and critically revised the manuscript. EE helped with the interpretation of data and critically revised the manuscript. $\mathrm{HO}$ helped with the interpretation of data and critically revised the manuscript. AG provided resources for the execution of this investigation and critically revised the manuscript. KW helped to analyze and interpret the data and revised the manuscript. $\mathrm{CH}$ helped to analyze and interpret the data and revised the manuscript. AS conceived the study design, selected studies, extracted and checked data and helped to write the manuscript. All authors read and approved the final manuscript.

Ethics approval and consent to participate

Not applicable.

Consent for publication

Not applicable.

\section{Competing interests}

The authors declare that they have no competing interests.

\section{Publisher's Note}

Springer Nature remains neutral with regard to jurisdictional claims in published maps and institutional affiliations.

\section{Author details}

1Department of Intensive Care, VU University Medical Center, De Boelelaan 1117, 1007, MB, Amsterdam, The Netherlands. ${ }^{2}$ Department of Internal Medicine, VU University Medical Center, Amsterdam, The Netherlands. ${ }^{3}$ Department of Physiology, VU University Medical Center, Amsterdam, The Netherlands. ${ }^{4}$ SYstematic Review Centre for Laboratory animal Experimentation (SYRCLE), Department for Health Evidence, Radboud Institute for Health Sciences, Radboud University Medical Center, Nijmegen, The Netherlands.

Received: 30 March 2018 Accepted: 9 July 2018

Published online: 04 August 2018

References

1. Cornet AD, Kooter AJ, Peters MJ, Smulders YM. The potential harm of oxygen therapy in medical emergencies. Crit Care. 2013;17:313.

2. Suzuki S, Eastwood GM, Peck L, Glassford NJ, Bellomo R. Current oxygen management in mechanically ventilated patients: a prospective observational cohort study. J Crit Care. 2013;28:647-54.

3. de Graaff AE, Dongelmans DA, Binnekade JM, de Jonge E. Clinicians' response to hyperoxia in ventilated patients in a Dutch ICU depends on the level of FiO2. Intensive Care Med. 2011;37:46-51.

4. Bitterman H, Brod V, Weisz G, Kushnir D, Bitterman N. Effects of oxygen on regional hemodynamics in hemorrhagic shock. Am J Physiol Heart Circ Physiol. 1996;271:H203-11.

5. Hauser B, et al. Hemodynamic, metabolic, and organ function effects of pure oxygen ventilation during established fecal peritonitis-induced septic shock. Crit Care Med. 2009;37:2465-9. 
6. Waisman D, et al. Effects of hyperoxia on local and remote microcirculatory inflammatory response after splanchnic ischemia and reperfusion. Am J Physiol Heart Circ Physiol. 2003;285:H643-52.

7. Halloran M, Donoghue E, Dainty C. Measurement of the retinal arteriolar response to a hyperoxic provocation in nonsmokers and smokers, using a high-resolution confocal scanning laser ophthalmoscope. J Biomed Opt. 2014;76012 https://doi.org/10.1117/1.JBO.19.7.076012.

8. Lott ME, et al. Impaired coronary and retinal vasomotor function to hyperoxia in individuals with type 2 diabetes. Microvasc Res. 2015;101:1-7.

9. Palkovits $\mathrm{S}$, et al. Retinal oxygen metabolism during normoxia and hyperoxia in healthy subjects. Invest Ophthalmol Vis Sci. 2014;55:4707-13.

10. Orbegozo Cortés $D$, et al. Normobaric hyperoxia alters the microcirculation in healthy volunteers. Microvasc Res. 2015;98:23-8.

11. Frisbee JC. Regulation of in situ skeletal muscle arteriolar tone: interactions between two parameters. Microcirculation. 2002;9:443-62.

12. Jackson WF. Arteriolar oxygen reactivity: where is the sensor? Am J Phys. 1987:253:H1120-6.

13. Messina EJ, Sun D, Koller A, Wolin MS, Kaley G. Increases in oxygen tension evoke arteriolar constriction by inhibiting endothelial prostaglandin synthesis. Microvasc Res. 1994;48:151-60.

14. Messmer C, Cabrales P, Intaglietta M. Effects of acute anemia and hyperoxia on oxygen distribution. Adv Exp Med Biol. 2012;737:213-8.

15. Rubanyi G, Paul RJ. O2-sensitivity of beta adrenergic responsiveness in isolated bovine and porcine coronary arteries. J Pharmacol Exp Ther. 1984; 230(3):692-8.

16. Smit B, et al. Hyperoxia does not directly affect vascular tone in isolated arteries from mice. PLoS One. 2017;12(8):e0182637.

17. Ngai JH, Roth PS, Paul RJ. Effects of endothelium on basal tone and agonist and O2 sensitivity in porcine coronary artery. J Pharmacol Exp Ther. 1990; 230(3):1053-9.

18. Rubanyi G, Paul RJ. Two distinct effects of oxygen on vascular tone in isolated porcine coronary arteries. Circ Res. 1985;56:1-10.

19. Jackson WF. Arteriolar oxygen reactivity: where is the sensor and what is the mechanism of action? J Physiol. 2016;594:5055-77.

20. Moher D, Liberati A, Tetzlaff J, A. D. PRISMA 2009 flow diagram. The PRISMA statement. 2009;6:1000097.

21. de Vries RBM, et al. A protocol format for the preparation, registration and publication of systematic reviews of animal intervention studies. EvidenceBased Preclin Med. 2015;2:e00007.

22. de Vries RBM, Hooijmans CR, Tillema A, Leenaars M, Ritskes-Hoitinga M. A search filter for increasing the retrieval of animal studies in Embase. Lab Anim. 2011:45:268-70.

23. Hooijmans CR, Tillema A, Leenaars M, Ritskes-Hoitinga M. Enhancing search efficiency by means of a search filter for finding all studies on animal experimentation in PubMed. Lab Anim. 2010:44:170-5.

24. Gibbons RD, Hedeker DR, Davis JM. Estimation of effect size from a series of experiments involving paired comparisons. J Educ Stat. 1993;18:271-9.

25. Borenstein $M$, Hedges LV, Higgins JPTR. Effect sizes based on means. In: Introduction to meta-analysis: John Wiley \& Sons, Ltd. p. 21-32. https://doi. org/10.1002/9780470743386.ch4.

26. Huedo-Medina TB, Sánchez-Meca J, Marín-Martínez F, Botella J. Assessing heterogeneity in meta-analysis: Q statistic or $\mathrm{I}^{2}$ index? Psychol Methods. 2006;11:193-206.

27. Crippa A, Orsini N. Dose-response meta-analysis of differences in means. BMC Med Res Methodol. 2016;16:91.

28. Akaike H. A new look at the statistical model identification. IEEE Trans Automat Contr. 1974;19:716-23.

29. Duval S, Tweedie R. Trim and fill: a simple funnel-plot-based method of testing and adjusting for publication bias in meta-analysis. Biometrics. 2000;56:455-63.

30. Zwetsloot PP, et al. Standardized mean differences cause funnel plot distortion in publication bias assessments. Elife. 2017;6

31. Frisbee JC, Lombard JH. Parenchymal tissue cytochrome P450 4A enzymes contribute to oxygen-induced alterations in skeletal muscle arteriolar tone Microvasc Res. 2002:63:340-3.

32. Jackson WF. Prostaglandins do not mediate arteriolar oxygen reactivity. Am J Phys. 1986;250:H1102-8.

33. Rafi JA, Boegehold MA. Microvascular responses to oxygen and muscle contraction in hypertensive dahl rats. Int J Microcirc Clin Exp. 1993;13:83-97.

34. Sauls BA, Boegehold MA. Arteriolar wall $P O(2)$ and nitric oxide release during sympathetic vasoconstriction in the rat intestine. Am J Physiol Heart Circ Physiol. 2000;279:H484-91.
35. Sauls BA, Boegehold MA. Adenosine linking reduced $\mathrm{O} 2$ to arteriolar NO release in intestine is not formed from extracellular ATP. Am J Physiol Heart Circ Physiol. 2001;281:H1193-200

36. Sullivan SM, Johnson PC. Effect of oxygen on arteriolar dimensions and blood flow in cat sartorius muscle. Am J Phys. 1981;241:H547-56.

37. Sakai N, Mizuno R, Ono N, Kato H, Ohhashi T. High oxygen tension constricts epineurial arterioles of the rat sciatic nerve via reactive oxygen species. Am J Physiol Heart Circ Physiol. 2007;293:H1498-507.

38. Duling BR. Oxygen sensitivity of vascular smooth muscle. II. In vivo studies. Am J Phys. 1974;227:42-9.

39. Milstein DMJ, et al. Sublingual microvascular perfusion is altered during normobaric and hyperbaric hyperoxia. Microvasc Res. 2016;105:93-102.

40. Ngo AT, Riemann M, Holstein-Rathlou N-H, Torp-Pedersen C, Jensen LJ. Significance of K(ATP) channels, L-type $\mathrm{Ca}^{2+}$ channels and CYP450-4A enzymes in oxygen sensing in mouse cremaster muscle arterioles in vivo. BMC Physiol. 2013;13:8

41. Ngo AT, Jensen $L$, Riemann $M$, Holstein-Rathlou N-H, Torp-Pedersen C. Oxygen sensing and conducted vasomotor responses in mouse cremaster arterioles in situ. Pflugers Arch. 2010;460:41-53.

42. Riemann $M$, et al. Oxygen-dependent vasomotor responses are conducted upstream in the mouse cremaster microcirculation. J Vasc Res. 2010:48:79-89.

43. Bertuglia S, Colantuoni A, Coppini G, Intaglietta M. Hypoxia- or hyperoxiainduced changes in arteriolar vasomotion in skeletal muscle microcirculation. Am J Physiol Heart Circ Physiol. 1991;260:H362-72.

44. Cabrales P, Tsai AG, Intaglietta M. Nitric oxide regulation of microvascular oxygen exchange during hypoxia and hyperoxia. J Appl Physiol. 2006;100: 1181-7.

45. Tsai AG, Cabrales P, Winslow RM, Intaglietta M. Microvascular oxygen distribution in awake hamster window chamber model during hyperoxia. Am J Physiol Heart Circ Physiol. 2003;285:H1537-45.

46. Kalsner S. Intrinsic prostaglandin release. A mediator of anoxia-induced relaxation in an isolated coronary artery preparation. Blood Vessels. 1976;13: 155-66.

47. Fredricks KT, Liu Y, Lombard JH. Response of extraparenchymal resistance arteries of rat skeletal muscle to reduced PO2. Am J Phys. 1994:H706-15.

48. Frisbee JC, Krishna UM, Falck JR, Lombard JH. Role of prostanoids and 20HETE in mediating oxygen-induced constriction of skeletal muscle resistance arteries. Microvasc Res. 2001;62:271-83.

49. Frisbee JC, Maier KG, Falck JR, Roman RJ, Lombard JH. Integration of hypoxic dilation signaling pathways for skeletal muscle resistance arteries. Am J Physiol Regul Integr Comp Physiol. 2002;283:R309-19.

50. Liu Y, Fredricks KT, Roman RJ, Lombard JH. Response of resistance arteries to reduced $\mathrm{PO} 2$ and vasodilators during hypertension and elevated salt intake. Am J Phys. 1997;273:H869-77.

51. Day RW, Lai WW, Klitzner TS, Ignarro L. Oxygen-mediated regulation of neonatal and adult rabbit aortic tension. Dev Pharmacol Ther. 1992:90-8.

52. Kwan YW, Wadsworth RM, Kane KA. Effects of hypoxia on the pharmacological responsiveness of isolated coronary artery rings from the sheep. Br J Pharmacol. 1989:96:849-56.

53. Pasgaard T, et al. Hyperoxia reduces basal release of nitric oxide and contracts porcine coronary arteries. Acta Physiol (Oxf). 2007:191:285-96.

54. Chang AE, Detar R. Oxygen and vascular smooth muscle contraction revisited. Am J Physiol. 1980:238:H716-28.

55. Pittman RN, Graham BA. Sensitivity of rabbit aorta to altered oxygen tension. Adv Exp Med Biol. 1986;200:373-83.

56. Vallet B, Winn MJ, Asante NK, Cain SM. Influence of oxygen on endothelium-derived relaxing factor/nitric oxide and $\mathrm{K}(+)$-dependent regulation of vascular tone. J Cardiovasc Pharmacol. 1994;24:595-602.

57. Lombard $\mathrm{JH}$, Stekiel WJ. Effect of oxygen on arteriolar diameters in the rat mesoappendix. Microvasc Res. 1985;30:346-9.

58. Dewhirst MW, et al. Arteriolar oxygenation in tumour and subcutaneous arterioles: effects of inspired air oxygen content. Br J Cancer Suppl. 1996:S241-6.

59. Jackson WF, Duling BR. The oxygen sensitivity of hamster cheek pouch arterioles. In vitro and in situ studies. Circ Res. 1983:53:515-25.

60. Welsh DG, Jackson WF, Segal SS. Oxygen induces electromechanical coupling in arteriolar smooth muscle cells: a role for L-type Ca2+ channels. Am J Phys. 1998;274:H2018-24

61. Gorczynski RJ, Duling BR. Role of oxygen in arteriolar functional vasodilation in hamster striated muscle. Am J Phys. 1978;235:H505-15.

62. Jackson WF. Lipoxygenase inhibitors block $\mathrm{O} 2$ responses of hamster cheek pouch arterioles. Am J Phys. 1988;255:H711-6. 
63. Jackson WF. Arteriolar oxygen reactivity is inhibited by leukotriene antagonists. Am J Phys. 1989;257:H1565-72.

64. Jackson WF. Regional differences in mechanism of action of oxygen on hamster arterioles. Am J Phys. 1993;265:H599-603.

65. Drenjancevic-Peric I, Greene AS, Kunert MP, Lombard JH. Arteriolar responses to vasodilator stimuli and elevated $\mathrm{P}(\mathrm{O} 2)$ in renin congenic and dahl salt-sensitive rats. Microcirculation. 2004;11:669-77.

66. Lombard JH, Stekiel WJ. Active tone and arteriolar responses to increased oxygen availability in the mesoappendix of spontaneously hypertensive rats. Microcirc Endothel. Lymphat. 1988:339-53.

67. Jackson WF. Nitric oxide does not mediate arteriolar oxygen reactivity. Microcirc Endothel Lymphat. 1991;7:199-215.

68. Komori M, Takada K, Ozaki M. Effects of inspired oxygen concentration on peripheral microcirculation studied by the rabbit ear chamber method. In Vivo. 2001;15:303-8.

69. Frisbee $\mathrm{JC}$, Lombard $\mathrm{JH}$. Elevated oxygen tension inhibits flow-induced dilation of skeletal muscle arterioles. Microvasc Res. 1999:58:99-107.

70. Hedegaard ER, Stankevicius E, Simonsen U, Fröbert O, Frobert O. Nonendothelial endothelin counteracts hypoxic vasodilation in porcine large coronary arteries. BMC Physiol. 2011;11:8.

71. Liu $X$, et al. Oxygen regulates the effective diffusion distance of nitric oxide in the aortic wall. Free Radic Biol Med. 2010;48:554-9.

72. Kunert MP, Roman RJ, Falck JR, Lombard JH. Differential effect of cytochrome P-450 omega-hydroxylase inhibition on O2-induced constriction of arterioles in SHR with early and established hypertension. Microcirculation. 2001:435-43. https://doi.org/10.1038/sj/mn/7800114.

73. Kunert MP, Roman RJ, Alonso-Galicia M, Falck JR, Lombard JH. Cytochrome P-450 omega-hydroxylase: a potential $\mathrm{O}(2)$ sensor in rat arterioles and skeletal muscle cells. Am J Physiol Heart Circ Physiol. 2001;280:H1840-5.

74. Macica C, Balazy M, Falck JR, Mioskowski C, Carroll MA. Characterization of cytochrome P-450-dependent arachidonic acid metabolism in rabbit intestine. Am J Phys. 1993;265:G735-41.

75. Rousseau A, Steinwall I, Woodson RD, Sjöberg F. Hyperoxia decreases cutaneous blood flow in high-perfusion areas. Microvasc Res. 2007;74:15-22.

76. Stub D, et al. Air versus oxygen in ST-segment elevation myocardial infarction. Circulation. 2015;131:2143-50.

77. Guensch DP, Fischer K, Shie N, Lebel J, Friedrich MG. Hyperoxia exacerbates myocardial ischemia in the presence of acute coronary artery stenosis in swine. Circ Cardiovasc Interv. 2015;8:e002928.

78. Meier J, et al. Regional blood flow during hyperoxic haemodilution. Clin Physiol Funct Imaging. 2005;25:158-65.

79. Sukhotnik I, et al. The effect of $100 \%$ oxygen on intestinal preservation and recovery following ischemia-reperfusion injury in rats. Crit Care Med. 2009; 37:1054-61.

80. Ishikawa $\mathrm{K}$, et al. Coronary artery constriction by oxygen breathing in patients with coronary disease. Tohoku J Exp Med. 1981;134:265-71.

81. Ishikawa K, Hayashi T, Tashi M. Reduction in diameter of coronary arteries by oxygen breathing in patients with coronary artery disease. Med.J Kinki Univ. 1978:7-13.

82. Gao Z, et al. Vitamin C prevents hyperoxia-mediated coronary vasoconstriction and impairment of myocardial function in healthy subjects. Eur J Appl Physiol. 2012;112:483-92.

83. McNulty PH, et al. Effects of supplemental oxygen administration on coronary blood flow in patients undergoing cardiac catheterization. AJP Hear Circ Physiol. 2005;288:H1057-62.

84. McNulty PH, et al. Effect of hyperoxia and vitamin C on coronary blood flow in patients with ischemic heart disease. J Appl Physiol. 2007;102:2040-5.

85. Davis MJ, Joyner WL, Gilmore JP. Microvascular pressure distribution and responses of pulmonary allografts and cheek pouch arterioles in the hamster to oxygen. Circ Res. 1981:125-32.

86. Lombard JH, Kaminski RP, Stekiel WJ. Arteriolar responses to changes in oxygen availability following single withdrawal hemorrhage. Microvasc Res. 1981:332-42. https://doi.org/10.1016/0026-2862(81)90016-9.

87. Taguchi A. Microcirculation during inhalation of $100 \%$ oxygen in rabbits. Masui. 1992:41:67-71.

88. Pries AR, Heide J, Ley K, Klotz KF, Gaehtgens P. Effect of oxygen tension on regulation of arteriolar diameter in skeletal muscle in situ. Microvasc Res. 1995:49:289-99.

89. Harder DR, et al. Identification of a putative microvascular oxygen sensor. Circ Res. 1996;79:54-61.
90. Lombard JH, et al. Cytochrome P-450 omega-hydroxylase senses $\mathrm{O} 2$ in hamster muscle, but not cheek pouch epithelium, microcirculation. Am J Phys. 1999;276:H503-8.

91. Frisbee $\mathrm{JC}$, Lombard $\mathrm{JH}$. Increased intravascular pressure does not enhance skeletal muscle arteriolar constriction to oxygen or angiotensin II. Microvasc Res. 2000;59:176-80.

92. Frisbee JC, Falck JR, Lombard JH. Contribution of cytochrome P-450 (omega)-hydroxylase to altered arteriolar reactivity with high-salt diet and hypertension. Am J Physiol Heart Circ Physiol. 2000;278:H1517-26.

93. Kunert MP, Friesma J, Falck JR, Lombard JH. CYP450 4A inhibition attenuates $\mathrm{O} 2$ induced arteriolar constriction in chronic but not acute Goldblatt hypertension. Microvasc Res. 2009;78:442-6.

94. Wang J, et al. Modulation of vascular $\mathrm{O} 2$ responses by cytochrome 450-4A omega-hydroxylase metabolites in dahl salt-sensitive rats. Microcirculation. 2009;16:345-54.
Ready to submit your research? Choose BMC and benefit from:

- fast, convenient online submission

- thorough peer review by experienced researchers in your field

- rapid publication on acceptance

- support for research data, including large and complex data types

- gold Open Access which fosters wider collaboration and increased citations

- maximum visibility for your research: over $100 \mathrm{M}$ website views per year

At BMC, research is always in progress.

Learn more biomedcentral.com/submissions 